\title{
Computer-based cognitive remediation program for the treatment of behavioral problems in children with intellectual disability: the «COGNITUS \& MOl» study protocol for a randomized controlled trial
}

\author{
Emilie Favre $^{1 *}$ (D) Elodie Peyroux ${ }^{1}$, Marie-Noelle Babinet ${ }^{1}$, Alice Poisson ${ }^{1}$ and Caroline Demily ${ }^{1,2}$
}

\begin{abstract}
Background: Comorbid psychiatric disorders are frequent in children with intellectual disability (ID). Given the limitations of drugs treatments, cognitive remediation could be a promising tool to reduce these challenging behaviors but evidence is still scarce. Our group recently developed the «COGNITUS \& MOl» program that is designed to train the attentional and visuospatial skills in children with ID. This study investigates the efficiency of the «COGNITUS \& MOl» program in this condition.

Methods: Children (age: 6.00-13.11) with mild to moderate ID and behavioral problems, will benefit from a therapy during a 16 week randomized controlled trial. One group will be randomly treated with the «COGNITUS \& MOl» program and the other with a motor skill and video viewing intervention. All participants will undergo a behavioral, functional and neurocognitive assessment at baseline, post-intervention, and 6-month follow-up. Primary outcome will be the change from the baseline of the score on the "hyperactivity - noncompliance" subscale of the Aberrant Behavior Checklist.
\end{abstract}

Discussion: If the results are conclusive, the «COGNITUS \& MOl» program could be added to the therapeutic arsenal against challenging behavior in children with ID.

Trial registration: ClinicalTrials NCT02797418. Date registered: 8th of June 2016.

Keywords: Cognitive remediation, Intellectual disability, Children, Challenging behaviour, Comorbid psychiatric disorder

\section{Background}

Intellectual disability (ID) is a neurodevelopmental medical condition defined by poor cognitive functioning and adaptive skills. It includes a lack of autonomy in daily life and weak adaptation to the environment. Comorbid psychiatric disorders frequently occur with ID [1-3]. Unfortunately, pharmacological treatments are often poorly effective and/or generate side effects [4-6]. Non-drug treatments could therefore be of particular interest. Among these approaches, cognitive remediation aims at developing strategies to

\footnotetext{
* Correspondence: emilie.favre@ch-le-vinatier.fr

${ }^{1}$ GenoPsy, Reference center for rare diseases with psychiatric symptoms, Centre Hospitalier le Vinatier and EDR-Psy team (CNRS UMR 5229 \& Lyon 1 University), 69678 BRON Cedex Lyon, Lyon, France

Full list of author information is available at the end of the article
}

overcome the daily impact of cognitive deficits [7] and could be a promising tool to reduce challenging behaviors in children with ID. Indeed, specific cognitive profiles - especially memory, attentional, visuospatial and executive deficits - are well documented in children with ID [8-10]. Impairment of these functions has been related to psychiatric and more generally to behavioral problems in other medical conditions [11, 12]. Moreover, attentional and visuospatial skills must underlie social cognition abilities, especially facial emotion recognition [13]. Impairment of social cognition frequently occurs in ID [14-16] and is associated with challenging behaviors $[16,17]$. The stimulating idea that comes out of these results is that appropriate cognitive training would allow an improvement in environmental stimuli processing and consequently in the behavioral response of children with ID. 
Despite these converging data, evidence-based non-drug treatments for behavioral problems in ID are still lacking. Previous studies have shown that cognitive remediation could improve cognitive abilities in children with ID [18-22] but - to our knowledge - few were interested in behavior and adaptive skills in daily life [23, 24].

Our group recently developed the «COGNITUS \& MOI» program, a cognitive remediation tool designed to train the attentional and visuospatial skills of 5-13 year-old children. COGNITUS is a friendly cartoon character that accompanies the child throughout various activities in paper-and-pencil, computerized, and at-home formats [25]. The «COGNITUS \& MOI» program could be of particular interest concerning challenging behaviors in children with ID for several reasons. First, the program is based on an active coaching approach and a flexible set of exercises that can be individually adapted according to the child's clinical profile. This is especially suitable in ID because of the high heterogeneity of clinical presentation. Second, this program focuses on visuospatial and attentional skills that could be closely related to behavioral disturbances. Therefore, the focus of this randomized controlled study is the "COGNITUS \& MOI» program in children with mild to moderate ID.

\section{Objectives of the study}

The primary objective of this study is to evaluate the effectiveness of the «COGNITUS \& MOI» program compared to a motor skill and video viewing intervention in terms of children's behavioral problems.

The secondary objectives are as follows:

(1) to investigate the effectiveness of the «COGNITUS \& MOI» program compared to a motor skill and video viewing intervention on children's neurocognitive abilities;

(2) to investigate the effectiveness of the "COGNITUS \& MOI» program compared to a motor skill and video viewing intervention on functional outcomes in children (adaptive behaviors, quality of life and self-esteem); and

(3) to evaluate the long-term maintenance (6 months after) of the beneficial effects of these treatments.

It is hypothesized that children who undergo the «COGNITUS \& MOI» program will demonstrate greater and more sustainable improvements in behavioral problems, neurocognitive abilities and functional outcomes than those who receive a motor skill and video viewing intervention.

\section{Methods and design Design}

Two groups of 100 children will benefit from a therapy during a 16 week randomized controlled trial. One group will be randomly treated with the "COGNITUS \& MOI» program and the other with a motor skill and video viewing intervention. In both groups, the preliminary session will consist of the presentation of the program to the child and their parents by the therapist. The following 16 therapy sessions will occur once a week and include paper-and-pencil, computer-based and at-home activities. All participants will undergo a behavioral, functional and neurocognitive assessment by an experienced psychologist. The assessment will occur before, immediately after and 6 months after the end of the program (baseline, post-treatment and 6-month follow-up). Figure 1 shows a schematic overview of the design of the study.

\section{Inclusion and exclusion criteria}

Children from 6 to 13 years old with mild to moderate intellectual disability in line with DSM-V criteria and behavioral problems are eligible for this study. Detailed inclusion and exclusion criteria are summarized in Table 1. Pychotropic medications and occupational, speech, orthoptic and/or psychomotor therapies should remain unchanged thorough the protocol. The intervention will be discontinued in case of clinical aggravation, patient's refusal to continue or after more than two unjustified absence rated by therapist.

\section{Recruitment}

Recruitment will occur among 6 French clinical genetic, psychiatric, or neurologic departments of public hospitals. Detailed oral and written information about the study will be given to the child and their parents by the investigator. For ethical reasons, parents will be informed that children allocated to the motor skill and video viewing intervention could benefit from the "COGNITUS \& MOI» program at the end of the study. Inclusion and exclusion criteria will be carefully verified by medical investigators. Parents will give written informed consent, and children will provide oral consent before inclusion in the research program.

\section{Randomization}

Four strata will be constructed based on age (6-9 and 10-13 years) and total Intellectual Quotient (IQ) score (45-55 and 56-70) to balance characteristics between the treatment groups. After the inclusion of a child, randomization will be done by the statistician involved in the research project and provided to each therapist. The participants and their parents will rate several measures - including primary outcome measures - and will be informed of their group assignment. However, the psychologist will blindly assess the participant's behavior, cognitive abilities and functional outcomes at baseline, post-treatment, and the 6-month follow-up. 


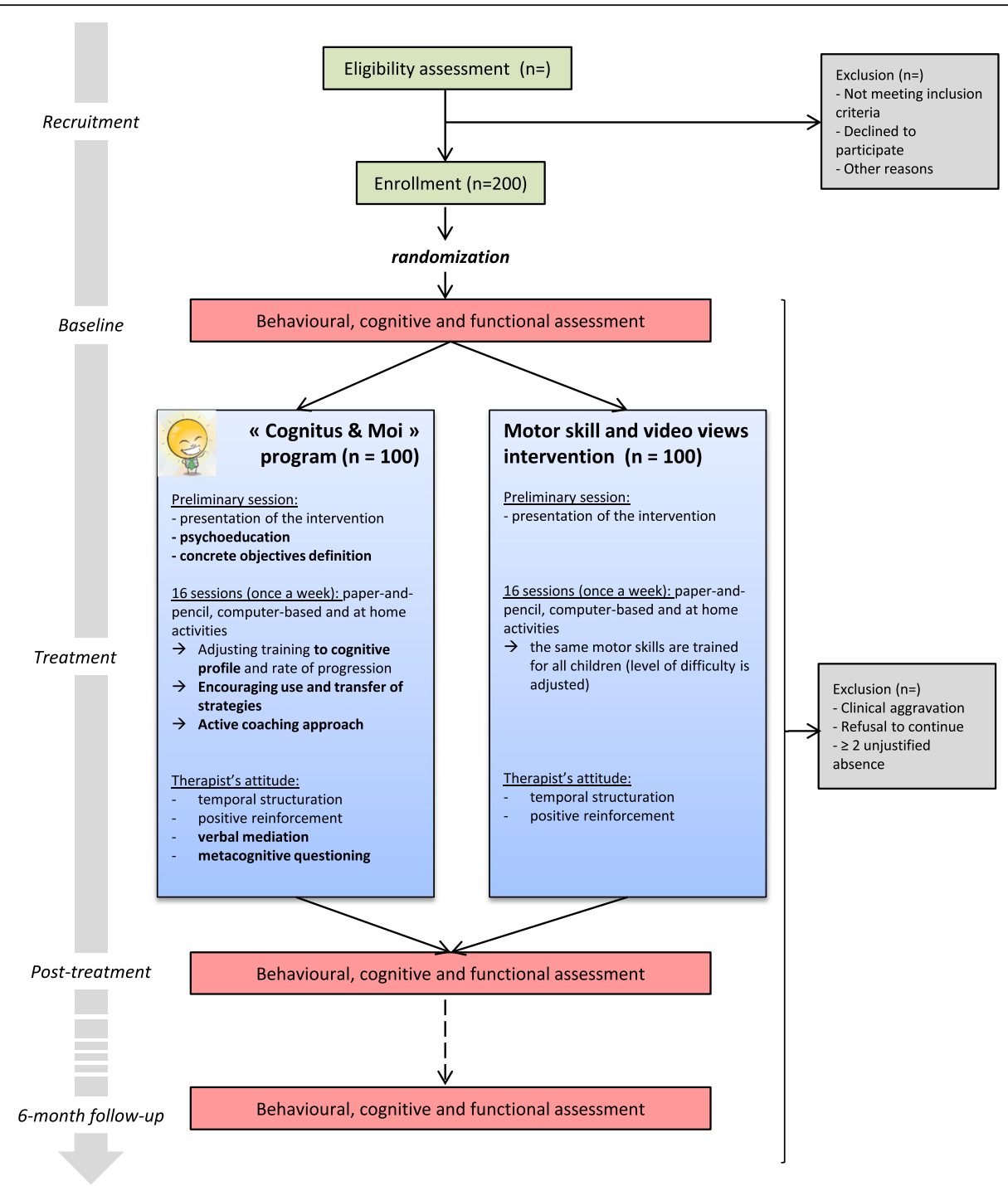

Fig. 1 Schematic overview of the «COGNITUS \& MOl» study protocol

Table 1 Inclusion and exclusion criteria

\begin{tabular}{ll}
\hline Inclusion criteria & Exclusion criteria
\end{tabular}

- age between 6 years 0 month and 13 years 11 months

- ABC scale total score equal or higher than 15

- total IQ score between 45 and 70 (WPPSI-III, WPPSIIIV, WISC-IV or WISC-V scale)

AND standard score of at least one domain of the VABS-II between 40 and 70

AND measurements carried out for less than 18 months

- French speaker

- Psychotropic medication remained unchanged for 1 month

- Occupational, speech, orthoptic and/or psychomotor therapies remained unchanged for 1 month

WPPSI Wechsler Preschool and Primary Scale of Intelligence (Wechsler, 2004; 2014), WISC Wechsler Intelligence Scale for Children (Wechsler, 2005; 2016), ABC Aberrant Behavior Checklist (Aman \& Singh, 1986), VABS-II Vineland Adaptive Behavior Scales - Second Edition (Sparrow, Cicchetti, \& Balla, 2015)
- DSM-5 criteria for autism spectrum disorder

- vascular, infectious or degenerative neurological condition

- medication for somatic problem that might interfere with brain functioning 
The «COGNITUS \& MOI» program

The «COGNITUS \& MOI» program is a cognitive remediation tool, specifically for children, and is dedicated to attentional and visuospatial functions. A precise description of the program has been previously published [25]. Briefly, it is based on the following four main principles:

(1) targeting daily life difficulties with concrete objectives;

(2) adjusting activities to the individual child's cognitive profile and rate of progression;

(3) encouraging the use and transfer of strategies while facing cognitive difficulties; and

(4) a coaching approach leading to an interactive process between the therapist, child and his or her parents.

During the program, the therapist resorts to strategies or attitudes such as temporal structuration, verbal mediation, positive reinforcement and above all metacognitive questioning.

The present protocol includes 17 meetings with a therapist that has been specifically trained in the «COGNITUS \& MOI» program. The first preliminary session will apply to the child and their parents. The therapist will do the following:

(1) go through the behavioral, neurocognitive and functional assessment and outline the strengths and weaknesses of the child's cognitive profile.

(2) provide psychoeducation by explaining to the child and their parents what attentional and visuospatial functions are and their implications in daily life. In particular, this is achieved through a comic strip given to the child and a brochure with written information given to the parents.

(3) define with the child and his parents the concrete aims of the therapy in accordance with his cognitive profile and difficulties in daily life.

(4) introduce the program.

(5) explain the role of at-home activities in strategy transfer and provide the child with the "COGNITUS \& MOI» book (a child-friendly notebook that holds reports of at-home activities).

Each of the following 16 weekly sessions will last 1 hour and be identically structured. Each session begins with the program description and a review of the at-home activity for the previous week. The paper-and-pencil activities and computerized activities occur next and take 15 min each. The therapist creates paper-and-pencil activities on a case-by-case basis depending on the child's cognitive profile and hobbies. The therapist chooses computerized activities from the exercises in the attentional and visuospatial modules. Each session ends with a Cognitus' facial expression recognition task and an explanation of the at-home activity to the child and their parents.

\section{The motor skill and video viewing intervention}

The motor skill and video viewing intervention is structured in a similar manner as the "COGNITUS \& MOI» program but does not follow the same key principles. Activities are fixed in advance and are not adjusted to the child's profile. Concrete objectives are not defined, and use of strategy is not encouraged. The therapist does not provide coaching except for positive reinforcement and temporal structuration of the sessions.

During the first session, the therapist will only explain the results of the assessment, introduce the program and provide the child with a child-friendly notebook that holds at-home activity reports.

The 16 weekly sessions will be structured in the same manner as the «COGNITUS \& MOI» program except that there will be no facial expression recognition task. The therapist will adjust the paper-and-pencil activities for difficulties but will conform to a specific order with regard to the fine motor functions that are taught in each session (spreading, tying, blowing, nailing, pouring, coloring, screwing, etc.). The computerized activities consist of passive viewing of videos that are appropriate for the child's age.

\section{Assessments and outcomes}

Assessments will include behavioral problems, neurocognitive abilities (language, memory, praxis and visuospatial abilities and attentional and executive functions), social cognitive processes (emotion recognition and theory of mind abilities) and functional outcome (quality of life, self-esteem and adaptive and daily living skills) measures. Assessments will involve the child, their parents and an experienced psychologist.

The primary outcome is the change from the baseline of the score on the "hyperactivity - noncompliance" subscale of the Aberrant Behavior Checklist [26].

Secondary outcomes are as follows:

(1) the change from the baseline of the scores for a semi-structured interview investigating adaptive behaviors and daily living skills,

(2) the change from the baseline of the scores of two questionnaires investigating quality of life and self-esteem,

(3) the change from the baseline of scores of paperand-pencil subtests, computerized subtests and questionnaires investigating praxis and visuospatial functions, memory, language, attentional and executive functions, and social cognition, and 
(4) the change of all scores from post-treatment to the 6-month follow up.

See Table 2 for a detailed overview of the assessments and outcome measures.

\section{Sample size}

To our knowledge, only one previous study has evaluated the effectiveness of a non-drug treatment using the "hyperactivity - noncompliance" score of the $\mathrm{ABC}$ as the primary outcome measurement [27]. A decrease of 3 points after treatment was found to be significant $(\mathrm{M}=12.1 ; \mathrm{SD}=8.9$ versus $M=9 ; \mathrm{SD}=7.4$ ). Therefore, we hypothesize that following the motor skills and video viewing intervention, we will observe a 3 point decrease of the "hyperactivity noncompliance" score. We expect that the decrease will be double for the "COGNITUS \& MOI» program: the difference between the two treatments would be at least 3 points. With an expected SD of 5, a power of 90\%, 1:1 allocation into the two groups, two-sided testing and a significance level of $5 \%$, a total sample of 126 participants is required. Given the insufficient information from the literature and the risk of dropout, 200 participants will be enrolled in the present study.

\section{Statistical analysis}

Descriptive statistics will be reported for each variable of interest for the two groups.

Table 2 Measurements

\begin{tabular}{|c|c|c|c|c|c|c|c|c|}
\hline & Kind of measure & Name & type & informant & recruitment & $\begin{array}{l}\text { baseline } \\
\text { assessment }\end{array}$ & $\begin{array}{l}\text { post-treatment } \\
\text { assessment }\end{array}$ & $\begin{array}{l}\text { 6-months follow up } \\
\text { assessment }\end{array}$ \\
\hline & IQ estimation & $\begin{array}{l}\text { WPPSI-III, WPPSI-IV, WISC-IV or } \\
\text { WISC-V }\end{array}$ & $P \& P$ & C & * & & & \\
\hline $\begin{array}{l}\text { primary } \\
\text { outcome }\end{array}$ & behavioural problems & $A B C$ & Q & $P$ & * & * & * & * \\
\hline \multirow[t]{22}{*}{$\begin{array}{l}\text { secondary } \\
\text { outcome }\end{array}$} & $\begin{array}{l}\text { adaptive and daily } \\
\text { living skills }\end{array}$ & VABS-II & $\begin{array}{l}\text { S } \\
\text { Int. }\end{array}$ & $P$ & * & * & * & * \\
\hline & quality of life & KIDSCREEN-27 & Q & $P$ & & * & * & * \\
\hline & self esteem & self-esteem subscale MDI-C & Q & C & & * & * & * \\
\hline & lexical knowledge & PPVT & $P \& P$ & C & & * & * & * \\
\hline & \multirow{2}{*}{$\begin{array}{l}\text { learning and long } \\
\text { term memory }\end{array}$} & word list CMS & $P \& P$ & C & & * & * & * \\
\hline & & dot locations CMS & $P \& P$ & C & & * & * & * \\
\hline & \multirow{5}{*}{$\begin{array}{l}\text { praxis and visuospatial } \\
\text { functions }\end{array}$} & visuomotor precision NEPSY-II & $P \& P$ & C & & * & * & * \\
\hline & & $\begin{array}{l}\text { imitating hand positions } \\
\text { NEPSY-II }\end{array}$ & $P \& P$ & C & & * & * & * \\
\hline & & arrows NEPSY-II & $P \& P$ & C & & * & * & * \\
\hline & & block construction NEPSY-II & $P \& P$ & C & & * & * & * \\
\hline & & route finding NEPSY-II & $P \& P$ & C & & * & * & * \\
\hline & \multirow[t]{4}{*}{ attentional functions } & sky search TEA-Ch & $P \& P$ & C & & * & * & * \\
\hline & & alertness TAP 2.3 & CT & C & & * & * & * \\
\hline & & auditory attention NEPSY-II & $P \& P$ & C & & * & * & * \\
\hline & & divided attention TAP 2.3 & CT & C & & * & * & * \\
\hline & \multirow[t]{4}{*}{ executive functions } & Go/NoGo TAP 2.3 & CT & C & & * & * & * \\
\hline & & response set NEPSY-II & $P \& P$ & C & & * & * & * \\
\hline & & inhibition NEPSY-II & $P \& P$ & C & & * & * & * \\
\hline & & mazes WISC-III & $P \& P$ & C & & * & * & * \\
\hline & \multirow[t]{3}{*}{ social cognition } & affect recognition NEPSY-II & $P \& P$ & C & & * & $*$ & * \\
\hline & & theory of mind NEPSY-II & $P \& P$ & C & & * & $*$ & * \\
\hline & & EQS & Q & $P$ & & * & * & * \\
\hline
\end{tabular}

WPPSI Wechsler Preschool and Primary Scale of Intelligence (Wechsler, 2004; 2014), WISC Wechsler Intelligence Scale for Children (Wechsler, 2005; 2016), ABC Aberrant Behavior Checklist (Aman \& Singh, 1986), VABS-II Vineland Adaptive Behavior Scales - Second Edition (Sparrow, Cicchetti, \& Balla, 2015), KIDSCREEN-27 (Robitail et al., 2007), MDI-C multiscore Depression Inventory for Children (Berndt \& Kaiser, 1999), PPVT Peabody Picture Vocabulary Test (Dunn, Thériault-Whalen, \& Dunn, 1993), CMS Children's Memory Scale (Cohen, 2001), NEPSY-Il Developmental NEuroPSYchological Assessment - Second Edition (Korkman, Kirk, \& Kemp, 2012), TEA-Ch Test of Everyday Attention for Children (Manly, Robertson, Anderson, \& Mimmo-Smith, 2004), TAP 2.3. Test of Attentional Performance - Version 2.3 (Zimmermann \& Fimm, 2012), WISC-III Wechsler Intelligence Scale for Children - Third Edition (Wechsler, 1996), EQS Empathy Quotient Questionnaire (Baron-Cohen $\&$ Wheelwright, 2004); $P \& P$ paper and pencil test; $Q$ questionnaire; $S$ Int. semi structured interview; $C T$ computerized test; $C$ child; $P$ parents 
First, independent-sample t-tests or non-parametric Mann-Whitney tests will be run to assess the lack of differences between groups in age, IQ or severity of $\mathrm{ABC}$ scores at baseline $(p<0.05$, two-sided).

Second, independent-sample t-tests or non-parametric Mann-Whitney tests will be run to compare the "hyperactivity - noncompliance" score change from the baseline to post-treatment assessment in the two groups $(p<0.05$, two-sided).

Third, similar tests will be run for each secondary outcome measure. The score changes will be separately considered from the baseline to post-treatment assessment and from the post-treatment to 6-month follow-up assessment ( $p<0.05$, two-sided).

\section{Discussion}

Drug treatments are often unsatisfactory methods to overcome challenging behaviors in children with mild to moderate ID. Cognitive remediation could be a promising alternative. To our knowledge, no program has been designed and validated for this clinical population. It is thus of great importance to undertake a treatment-efficacy study. The «COGNITUS \& MOI» study specifically investigates the efficiency of a cognitive remediation program designed for children with mild to moderate ID.

The primary strength of this study is the «COGNITUS \& MOI» program itself. It is of particular interest in ID considering that these patients have heterogeneous cognitive profiles, have few adaptive skills in daily life, and often feel incompetent. The «COGNITUS \& MOI» program allows for the precise adaptation to individual clinical and cognitive profiles. Furthermore, it is based on active coaching that favors positive reinforcement and metacognitive training. It aims at improving daily life by encouraging strategy transfer. The partnership with the parents must also be emphasized because it allows for the consideration of the context of the child's family unit. The randomized design is another strong point of the present study, highlighting the specific effect of the «COGNITUS \& MOI» program versus a nonspecific intervention. It is well-known that 'therapeutic alliance' explains a significant proportion of any behavioral therapy efficiency. Therefore, it is very important to differentiate these general effects to validate the «COGNITUS \& $\mathrm{MOI}$ » cognitive remediation program. The evaluation of the long-term maintenance of beneficial effects is also of particular relevance.

One limitation of this study may be that the primary outcome is assessed in an open-label fashion. Parents score their children using the $\mathrm{ABC}$ scale and know the intervention their child received. The use of this kind of measure is nevertheless fully justified by the fact that the study takes into account functional outcomes in everyday life, and parents receive standardized instructions to limit potential biases. Another limitation could concern the feasibility of the cognitive remediation program in children with ID. For example, it would be fair to question the use of metacognitive questioning with these children and their attendance throughout the program. Preliminary case studies confirm that the "COGNITUS \& MOI» program is fully adapted to this clinical population, even in the case of poor verbal expression abilities.

\section{Conclusion}

If the results are conclusive, the "COGNITUS \& MOI» program could be added to the therapeutic arsenal against challenging behavior in children with ID. This non-drug treatment would be of particular interest because of the minimal adverse effects, which are present with drug treatments. It might also be a favorable therapy throughout life because cognitive stimulation in children could help achieve developmental milestones. Another possibility is the evaluation of the "COGNITUS \& MOI» program in improving behavior and adaptive skills in adults with moderate ID, for which there are currently few adequate behavioral therapies.

\section{Abbreviations \\ ABC: Aberrant Behavior Checklist; ID: Intellectual Disability; IQ: Intellectual Quotient}

\section{Acknowledgements}

The authors thank Véronique Vial-Fallot and Lydie Sartelet for their contribution towards the article submission process. The authors wish to thank the children and families for participating in this study.

\section{Administrative information}

Protocol identifier: version 2, date: June 2017.

Trial sponsor: The research unit of the Centre Hospitalier Le Vinatier will manage monitoring of this study.

Correspondence: 95 boulevard Pinel - BP 30039 F-69678 BRON cedex, France, T: + 33,437,915,531, email: veronique.vial@ch-le-vinatier.fr.

Trial registration database: ClinicalTrials.gov.

Trial registration: NCT02797418.

Registration date: 08-06-2016.

\section{Funding}

This project is funded by a grant of the nonprofit Francophone Association of Cognitive Remediation (AFRC) and by a grant of the Scientific Committee of Research of the Hospital 'Le Vinatier' (CSR H05). The second funding source was involved in the design of this study. The funding sources will not have any role during its execution, analyses, interpretation of the data, or decision to submit results.

\section{Availability of data and materials}

Data sharing is not applicable to this article as no datasets were generated or analyzed. The "COGNITUS \& MOI" program is commercialized by Scientific Brain Training SA.

Authors' contributions

$E F, E P, M N B, A P$, and $C D$ wrote the current manuscript. MNB will be responsible for collecting the data. $C D$ is the lead author and principal investigator. All authors read and approved the final manuscript.

Ethics approval and consent to participate

We adhered to SPIRIT guidelines. This study was approved by the local ethic committee (decision of the Comité de Protection des Personnes SUD-EST IV of 6 April 2016; 1st amendment of 15 march 2017; 2nd amendment 27 
march 2018; ID-RCB: 2015-A00858-41) and was authorized by the French National Agency for Medicines and Health Products Safety (ANSM 160096B-31). All amendments will be reported to the ethic committee. Consent to participate will be obtained from all participants and their parents.

\section{Consent for publication}

Not applicable.

\section{Competing interests}

Scientific Brain Training SA provided substantial technical support to develop computerized activities of the "COGNITUS \& MOI" program. Authors declare that they have no other competing interests.

\section{Publisher's Note}

Springer Nature remains neutral with regard to jurisdictional claims in published maps and institutional affiliations.

\section{Author details}

${ }^{1}$ GenoPsy, Reference center for rare diseases with psychiatric symptoms, Centre Hospitalier le Vinatier and EDR-Psy team (CNRS UMR 5229 \& Lyon 1 University), 69678 BRON Cedex Lyon, Lyon, France. 'Faculty of Medicine "Lyon Sud Charles Meyrieux", Lyon 1 University, Lyon, France.

Received: 13 November 2017 Accepted: 9 July 2018

Published online: 20 July 2018

\section{References}

1. Einfeld SL, Ellis LA, Emerson E. Comorbidity of intellectual disability and mental disorder in children and adolescents: a systematic review. J Intellect Develop Disabil. 2011;36:137-43.

2. Linna SL, Moilanen I, Ebeling H, Piha J, Kumpulainen K, Tamminen T, et al. Psychiatric symptoms in children with intellectual disability. Eur Child Adolesc Psychiatry. 1999;8(Suppl 4):77-82.

3. Munir KM. The co-occurrence of mental disorders in children and adolescents with intellectual disability/intellectual developmental disorder. Curr Opin Psychiatry. 2016:29:95-102.

4. de Kuijper G, Hoekstra P, Visser F, Scholte FA, Penning C, Evenhuis H. Use of antipsychotic drugs in individuals with intellectual disability (ID) in the Netherlands: prevalence and reasons for prescription. J Intellect Disabil Res JIDR. 2010;54:659-67.

5. McQuire C, Hassiotis A, Harrison B, Pilling S. Pharmacological interventions for challenging behaviour in children with intellectual disabilities: a systematic review and meta-analysis. BMC Psychiatry. 2015;15:303.

6. Tsiouris JA. Pharmacotherapy for aggressive behaviours in persons with intellectual disabilities: treatment or mistreatment? J. Intellect. Disabil. Res. JIDR. 2010;54:1-16.

7. Ben-Yishay Y. Foreword. Neuropsychol Rehabil. 2008;18:513-21.

8. Danielsson H, Henry L, Rönnberg J, Nilsson L-G. Executive functions in individuals with intellectual disability. Res Dev Disabil. 2010;31:1299-304.

9. Merrill EC, O'dekirk JM. Visual selective attention and mental retardation. Cogn Neuropsychol. 1994;11:117-32.

10. Schuchardt K, Gebhardt M, Mäehler C. Working memory functions in children with different degrees of intellectual disability: working memory functions. J Intellect Disabil Res. 2010;54:346-53.

11. Çak HT, Çengel Kültür SE, Gökler B, Öktem F, Taşkıran C. The behavior rating inventory of executive function and continuous performance test in preschoolers with attention deficit hyperactivity disorder. Psychiatry Investig. 2017;14:260-70.

12. Vangkilde A, Jepsen JRM, Schmock H, Olesen C, Arnarsdóttir S, Baaré WFC, et al. Associations between social cognition, skills, and function and subclinical negative and positive symptoms in 22q11.2 deletion syndrome. J Neurodev Disord. 2016;8:42.

13. Thakkar KN, Brugger P, Park S. Exploring empathic space: correlates of perspective transformation ability and biases in spatial attention. PLoS One. 2009:4:e5864

14. Dodge KA, Pettit GS. A biopsychosocial model of the development of chronic conduct problems in adolescence. Dev Psychol. 2003;39:349-71.

15. Hetzroni $\mathrm{O}$, Oren B. Effects of intelligence level and place of residence on the ability of individuals with mental retardation to identify facial expressions. Res Dev Disabil. 2002;23:369-78.
16. van Nieuwenhuijzen $M$, de Castro BO, Wijnroks L, Vermeer A, Matthys W Social problem-solving and mild intellectual disabilities: relations with externalizing behavior and therapeutic context. Am J Intellect Dev Disabil. 2009:114:42-51.

17. Ellis CR, Lindstrom KL, Villani TM, Singh NN, Best AM, ASW W, et al. Recognition of facial expressions of emotion by children with emotional and behavioral disorders. J Child Fam Stud. 1997;6:453-70.

18. Bussy $G$, Rigard $C$, des Portes $V$. Impact d'un entrainement de la mémoire à court-terme verbale sur le langage d'enfants ayant une déficience intellectuelle. Rev Francoph Défic Intellect. 2013;24:141-51.

19. Comblain A. Working memory in Down syndrome: training the rehearsal strategy. Syndr Res Pract. 1994;2:123-6.

20. Danielsson H, Zottarel V, Palmqvist $L$, Lanfranchi $S$. The effectiveness of working memory training with individuals with intellectual disabilities - a meta-analytic review. Front Psychol. 2015;6:1230

21. Hulme C, Mackenzie S. Working Memory and Severe learning difficulties. Hove: Erlbaum; 1992.

22. Kendall C, Borkowski J, Cavanaugh J. Metamemory and the transfer of an interrogative strategy by EMR children. Intelligence. 1980;4:255-70.

23. Galbiati S, Recla M, Pastore V, Liscio M, Bardoni A, Castelli E, et al. Attention remediation following traumatic brain injury in childhood and adolescence. Neuropsychology. 2009;23:40-9.

24. Roording-Ragetlie S, Klip H, Buitelaar J, Slaats-Willemse D. Working memory training in children with neuropsychiatric disorders and mild to borderline intellectual functioning, the role of coaching; a double-blind randomized controlled trial. BMC Psychiatry [Internet]. 2017 [cited 2017 Jul 5];17:114. Available from: http://bmcpsychiatry.biomedcentral.com/articles/10.1186/ s12888-017-1274-6

25. Demily C, Rigard C, Peyroux E, Chesnoy-Servanin G, Morel A, Franck N. «COGNITUS \& MOI»: a computer-based cognitive remediation program for children with intellectual disability. Front Psychiatry. 2016;7:10.

26. Aman M, Singh N. Aberrant behavior checklist manual. East Aurora, NY: Slosson Publications; 1986.

27. Tse J, Strulovitch J, Tagalakis V, Meng L, Fombonne E. Social skills training for adolescents with Asperger syndrome and high-functioning autism. J Autism Dev Disord. 2007;37:1960-8.
Ready to submit your research? Choose BMC and benefit from:

- fast, convenient online submission

- thorough peer review by experienced researchers in your field

- rapid publication on acceptance

- support for research data, including large and complex data types

- gold Open Access which fosters wider collaboration and increased citations

- maximum visibility for your research: over $100 \mathrm{M}$ website views per year

At BMC, research is always in progress.

Learn more biomedcentral.com/submissions 